This is a postprint version of: González-albo, B.; Bordons, M. "Articles vs. Proceedings Papers: Do they differ in research relevance and impact? A case study in the Library and Information Science field."

Journal of Informetrics 5(3): 369-381, 2011. DOI: 10.1016/j.joi.2011.01.011

The final publication is available at ScienceDirect:

http://www.sciencedirect.com/science/article/pii/S1751157711000125

\title{
Articles vs. Proceedings Papers: Do they differ in research relevance and impact? A case study in the Library and Information Science field
}

\author{
Borja González-Albo ${ }^{a}$ * and María Bordons ${ }^{a}$ \\ ${ }^{a}$ Instituto de Estudios Documentales en Ciencia y Tecnología (IEDCYT), Center for Human and Social \\ Sciences (CCHS), Spanish National Research Council (CSIC), Albasanz 26-28, 28037 Madrid, Spain. \\ borja.gonzalezalbo@cchs.csic.es; maria.bordons@cchs.csic.es
}

*Corresponding author. E-mail: borja.gonzalezalbo@cchs.csic.es. Telephone Number: 0034916022893

\begin{abstract}
In 2008, the type of document "proceedings paper" (PP) was assigned in the WoS database to journal articles which were initially presented at a conference and later adapted for publication in a journal. Since the use of two different labels ("article" and "proceedings paper") might lead to infer differences in their relevance and/or quality, this paper presents a comparative study of standard journal articles and PP in journals to explore potential differences between them. The study focuses on the Library and Information Science field in the Web of Science database and covers the 1990-2008 period. PP approximately account for $9 \%$ of the total number of articles in this field, two-thirds of which are published in monographic issues devoted to conferences, which tend to be concentrated in specific journals. Proceedings papers emerge as an heterogeneous set comprising PP in ordinary issues, similar to standard articles in structure and impact of research; and PP in monographic issues, which seem to be less comprehensive and tend to receive less citations. Faster publication of PP in monographic than in ordinary issues may conceal differences in the review process undergone by either type of paper. The main implications of these results for authors, bibliometricians, journal editors and research evaluators are pointed out.
\end{abstract}

\section{Keyword}

Proceedings Papers; Document type; Conference; Library and Information Science; Web of Science

\section{Introduction}

Conferences play an important role in scholarly communication, since they provide scientists with an opportunity to present and discuss preliminary results of their research and improve their personal social networks. Moreover, conferences allow scientists to stay abreast of current research trends in their field and learn about cutting-edge developments in their speciality. Papers presented at a conference are frequently published as abstracts or as full papers in conference proceedings, and even extended to meet journal article requirements. Journal publication is sought because authors try to increase the visibility of their research. Although the dissemination of conference proceedings is being increasingly improved through collection in digital libraries, inclusion in bibliographic databases or repositories or even making them available at the conference website, it is still lower than that of journal articles. On the other hand, the higher weight assigned to journal articles as compared to proceedings papers in research assessment procedures in many disciplines is also an incitement for scientists to publish extended versions of their conference presentations in journals.

Conference proceedings and extended journal articles serve different and complementary functions in scientific communication. Conferences allow scientists to present initial research, collect feedback from colleagues and improve future research, while journal papers are usually 
more complete, correspond to a more mature report of the research and have some archival function (Bar-llan, 2010; Montesi and Mackenzie, 2008). In a study based on editor interviews and author questionnaires in the field of software engineering, Montesi and Mackenzie (2008) conclude that conference papers tend to be short and only contain the most exciting part of the research, while journal papers tend to be longer and may contain more detailed information allowing replication and full understanding of the results. Moreover, it could be the case that not all conference presentations are suitable for extension into an article. In some cases, they present hypotheses that cannot be empirically confirmed or they focus on the application of techniques instead of dealing with new discoveries and consequently such papers are not considered eligible for publication in a scholarly journal (although this type of presentation can be extremely useful for conference participants) (Drott, 1995; Montesi and Mackenzie, 2008). All in all, conference proceedings and extended journal articles are both valuable for scientific purposes, although, in many disciplines, the latter are deemed to be a more formal and less ephemeral research output.

The extension of conference presentations into journal articles is common practice in many disciplines, but on a different scale depending on the field. It accounted for $30-50 \%$ of conference papers in different medical subfields (Miguel-Dasit et al., 2006; Kho, 2009) and for a third in scientometrics (Aleixandre et al., 2009). Even in engineering, where scientific meetings are especially important for scholarly communication (Glanzel et al., 2005) and papers presented at conferences are deemed formal papers valid for assessing research and for tenure decisions (Montesi and Mackenzie, 2008), the practice of extending conference presentations into journal articles exists to some extent, probably to enhance the visibility and impact of the research. The fact that journal articles are more likely to be cited than conference papers (Lisée and Larivière, 2008; Goodrum et al., 2001) and that proceedings become obsolete faster than other document types could be the underlying reasons (Lisée and Larivière, 2008), since authors may tend to publish in journal articles to optimise their citation track. For instance, a third of conference papers analysed in a study on software engineering (Montesi et al., 2008) and a quarter of those produced by a sample of highly-cited scientists in computer science (BarIlan, 2010) were later published as journal articles.

In 2008, the type of document "proceedings paper" was included in the WoS database to design documents initially presented at a conference or workshop and later adapted for publication in a journal. Until 2008, these documents were considered as journal articles by the Web of Science. It is important to underline that "proceedings paper" is also the label assigned by Thomson Reuters to full papers recorded in conference proceedings in the Conference Proceedings Citation Index, now available together with the SCIE, SSCl and AHCl databases at the Web of Knowledge platform. Although Thomson Reuters states that "we are not in any way commenting on the scholarly status of these documents (proceedings papers in journals) in making this designation" (WoS, 2009), we consider that the use of these two different labels ("article" and "proceedings paper") applied to papers in journals could be deceptive and might lead to infer differences in their relevance and/or quality. As pointed out above, "conference papers" are usually considered as less elaborate and less mature products than articles. Can this view of a "less elaborate product" be extended to the type of document identified by the WoS as "proceedings paper"? If so, it may even have some bearing on the way they are considered in research evaluation processes. Under this assumption, the objective of this paper is to conduct a comparative study of standard articles and proceedings papers in journals to explore potential differences and similarities as regards their structure and relevance in the field of Library and Information Science ${ }^{1}$.

The following questions are addressed: What is the proportion of proceedings papers in this field? What are the observed trends over the years? Do proceedings papers tend to be concentrated in specific journals or are they spread over a diversified range of publications? And finally and more important, are there differences between proceedings papers and standard articles in their structural features and impact?

\section{Methods}

The study focuses on the field of Library and Information Science (LIS), defined according to the Web of Science classification of journals into subject categories. A total of 70 journals were 
analysed. Citable items (articles, reviews, notes and proceedings papers) published in Library and Information Science (LIS) journals during the 1990-2008 period were downloaded from the Web of Science database ${ }^{2}$.

The comparative study of articles and proceedings papers (PP) was based on the analysis of the following aspects of documents:

a) Structural features of research. The number of authors, centres, references and pages are analysed as indirect indicators of the complexity and completeness of the research. Collaboration between different authors and centres may increase the difficulties in conducting the research, but also its scope and final results. Collaboration is beneficial for individual scientists and for scientific progress in general (Bordons and Gómez, 2000). On the other hand, a higher number of references and pages can be associated to more comprehensive papers (McVeigh and Mann, 2009), and they are more likely to produce some impact on the community, i.e. to attract a higher number of citations (Haslman et al., 2008).

b) Relevance of papers. The number of citations received by papers from the publication year to the downloading date (for all journals) with a three-year citation window (for the journals with a higher number of PP, hereinafter referred to as "core journals") is calculated as an indicator of the impact of the research. The use of citations as an indicator of the impact of research over the scientific community is widespread in the literature (Moed, 2005).

Could we expect a lower quality of PP as compared to articles? In principle, this is not expected, since PP are evaluated by experts before being accepted for publication. In our opinion, a good evaluation of papers is a filter against the publication of low quality documents. However, if the evaluation of PP were less rigorous and more superficial than that of standard articles, it could lead to lower-quality documents which in the long term might attract fewer citations than standard articles. Accordingly, the following question was addressed: Do PP receive fewer citations than standard articles? (Question 1).

It is common knowledge that some journals publish special monographic issues devoted to a particular conference. These documents are usually subject to a refereeing process, but it is sometimes independent from the standard evaluation process developed by the journal. Members of the scientific committee of the conference can also play the role of advisers for the evaluation of the conference proceedings. Could it be the case that this evaluation were less strict that the standard one at the journal? To address this issue in our study, PP were classified in two different subtypes: PP in monographic issues (PPm) and PP in ordinary issues (PPo) and the following question was raised: do PPm and $P P o$ differ in their structure and impact? (Question 2). For the purposes of this study, issues with more than $75 \%$ of PP among their papers were labelled as "monographic issues". It should be noted that "monographic issues" do not always correspond with the "supplements" of the journals, since we have observed that supplements do not exclusively contain PP and that PP can be published in regular journal issues too. According to our definition of PPm, around $70 \%$ of them were published in supplements.

Additionally, we hypothesize that the times span between the holding of the conference and the publication of the related document could give us some information about the rigour of the review process. It is clear that a long time span does not guarantee a rigorous review of documents, but we consider that a very fast publication of documents after the holding of the conference might suggest a poor or even a non-existent evaluation process. We consider that the whole process comprising the submission of the article by the authors, the assessment of manuscripts by peers and the submission of the final article by the authors (once the changes suggested by the peer-reviewers have been introduced) needs some time to be completed. So we explored the following: Are there differences between PP in ordinary and monographic issues in the time span between the holding of the conference and the publication of the related document? (Question 3). 
The structure and impact of articles and PP are explored through univariate statistical analysis (comparison of means for non-parametric distributions) but also with a multivariate approach (categorical principal component analysis) (SPSS, version 17). Categorical principal component analysis enables us to obtain a global analysis of data. This technique is used to extend standard principal component analysis to categorical variables (e.g. ordinal) with the advantage that it does not assume either linear relationship among numeric data or multivariate normal data. Categorical variables are transformed by assigning optimal scale values to the categories, resulting in numeric-valued transformed variables. A detailed description of the technique can be found in Meulman and Heiser (2007).

The paper structure is as follows. Firstly, general data concerning main features of the distribution of proceedings papers by years and journals are shown. Secondly, differences in the structure and relevance of articles and proceedings published in ordinary and monographic issues of journals are analysed. The first part of the study considers all publications, while the second part focuses on "core journals", which concentrate $66 \%$ of the proceedings papers. Finally, the relationship between the different variables analysed is studied from a global perspective by means of categorical principal component analysis.

\section{Results}

During the 1990-2008 period, a total of 31,865 articles and 3,151 proceedings papers were identified in Library and Information Science (WoS category) and published in 70 different journals. Proceedings papers represent $9 \%$ of this output.

The evolution of the number of proceedings papers over the years was irregular. It accounted for $3 \%$ of the output in 1990, peaked in $1994(24 \%)$ and $1999(21 \%)$, and declined afterwards, accounting for $4 \%$ of the output in 2008 (Figure 1 -PP broken down into monographic and ordinary issues-). Peaks were related with the publication of monographic issues devoted to specific conferences.

Figure 1. Annual evolution of citable items in Library and Information Science (WoS, 1990-2008)

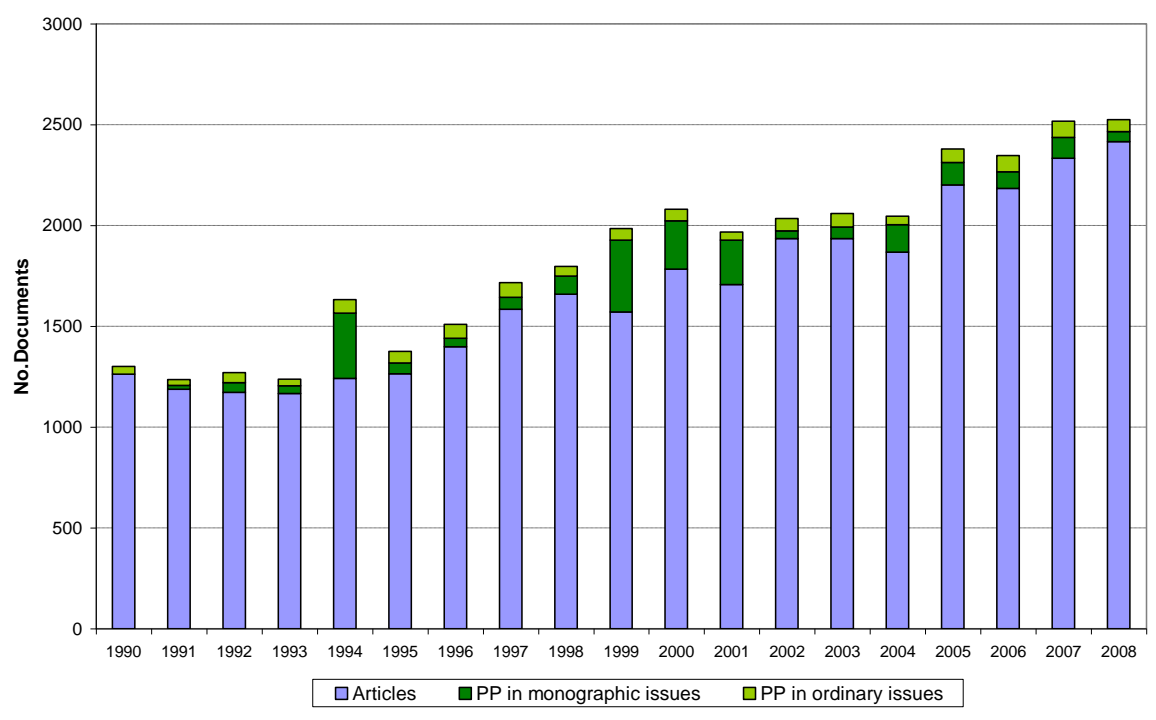

Note: the first dimension displays $88 \%$ of the inertia.

\subsection{PP by journal}

Two journals concentrate a large amount of the total PP: Journal of the American Medical Informatics Association (JAMIA) (961 PP, 30.5\% of the total) and Scientometrics (371 PP, $11.8 \%)$. 
The percentage of PP within each journal varies significantly from one journal to another, ranging from $0 \%$ to $48 \%$ of total articles (see Annex 1). Around $11 \%$ of journals did not have any PP during the period while in $54 \%$ of the journals PP represent less than $10 \%$ of their articles. Among the journals with the highest percentage of PP we can mention JAMIA (48\% of PP), Research Evaluation (43\%), Information Research (33\%), Information Technology and Management (30\%) (see Annex 1). The percentage of PP in Scientometrics is lower (around $20 \%$ ) because although it is the second most productive journal of PP in absolute numbers, it has a large overall number of documents.

Table 1. Distribution of journals by their percentage of proceedings papers in the 1990-2008 period

\begin{tabular}{crr}
\hline$\%$ PP/journal & No.Journals & \% Journals \\
\hline $0 \%$ & 8 & 11.43 \\
$>0 \%-10 \%$ & 38 & 54.29 \\
$>=10 \%-20 \%$ & 18 & 25.71 \\
$>=20 \%-30 \%$ & 2 & 2.86 \\
$>=30 \%$ & 4 & 5.71 \\
\hline Total & 70 & \\
\hline
\end{tabular}

Do journals publish special issues covering the material presented at a specific meeting? Or do PP coexist with standard articles in a given issue? Our data show that almost $19 \%$ of the issues with any PP were "monographic issues", assuming that a monographic issue is that with more than $75 \%$ of PP among its papers.

\subsection{PP in monographic and ordinary issues}

Considering all the PP, 2,074 (66\%) were published in monographic issues (PPm) and 1,077 $(34 \%)$ in ordinary ones (PPo). It is interesting to note that PPo were more evenly distributed over journals than PPm. As shown in figure 2, three journals accounted for approximately $60 \%$ of the PP in the case of monographic issues while 16 journals were needed to account for this percentage of PPo. The journals that concentrate the highest number of PPm are JAMIA and Scientometrics.

Figure 2. Cumulative distribution of PPo and PPm by journal

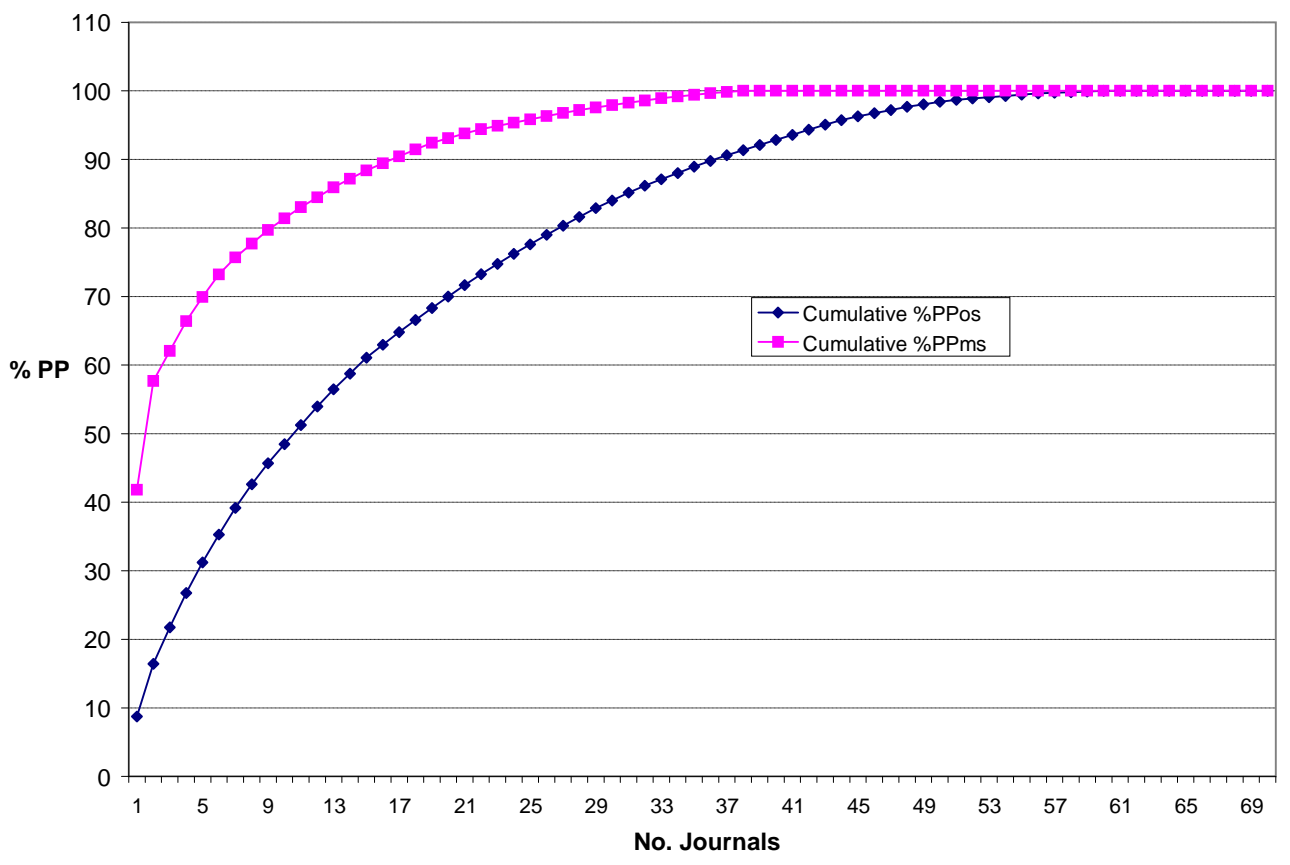


In order to obtain a general overview of the field, it is useful to classify journals according to their percentage distribution of articles, PP in monographic issues and PP in ordinary issues. By means of hierarchical clustering (Ward method, squared Euclidian distance) we obtain three different clusters or groups of journals with increasing presence of PP. The first cluster includes 39 journals in which articles constitute on average more than $98 \%$ of the documents and very few PPm are observed (less than 1\%). The second cluster comprises 27 journals in which articles account for around $88 \%$ of documents and there is a small presence of PPo and PPm (around $6 \%$ of each). The third cluster only includes 4 journals, which publish on average around $61 \%$ of articles and a very large number of PPm (34\% of total articles) (Table 2). The journals included in the third cluster are the following: Information Research, Information Technology \& Management, Journal of the American Medical Informatics Association, and Research Evaluation.

Table 2. Description of the journal clusters by distribution of PP

\begin{tabular}{lccccc}
\hline & $\mathrm{N}$ & $\begin{array}{c}\text { \% PP } \\
\text { monographic } \\
\text { issues }\end{array}$ & $\begin{array}{c}\text { \% PP } \\
\text { ordinary } \\
\text { issues }\end{array}$ & $\begin{array}{c}\% \\
\text { Articles }\end{array}$ & Description \\
\hline Cluster 1 & 39 & $0.47(0.95)$ & $1.91(1.82)$ & $97.63(2.12)$ & Almost no PP \\
Cluster 2 & 27 & $6.59(4.57)$ & $5.75(3.97)$ & $87.66(3.87)$ & Low presence of PP \\
Cluster 3 & 4 & $34.27(6.97)$ & $4.32(3.10)$ & $61.41(8.44)$ & High presence of PP \\
\hline Total & 70 & $4.76(8.53)$ & $3.53(3.41)$ & $91.71(9.52)$ & \\
\hline \multicolumn{4}{l}{ Note: Data expressed as average (standard deviation) }
\end{tabular}

Note: Data expressed as average (standard deviation)

Figure 3 is obtained using correspondence analysis with exploratory purposes, locating journals according to their document type pattern. A triangle marks the document types (articles, PPm, PPo), while a circle is used for journals. The first dimension separates journals with a high share of PP in monographic issues (right side of the plot) from the rest. The journals with the highest rate of PP in monographic issues are JAMIA, Information Technology \& Management, Information Research and Research Evaluation (Cluster 3). The second dimension separates journals with a high share of PP in ordinary issues (bottom of the plot) from the rest of the journals. Thus some journals such as Social Science Information sur les Sciences Sociales or Malasyan Journal of Library \& Information Science are located at the bottom of the chart (Cluster 2). The chart's centre concentrates most of the journals, in which articles are the most frequent type of document (Cluster 1). 
Figure 3. Correspondence analysis of journals by document type pattern

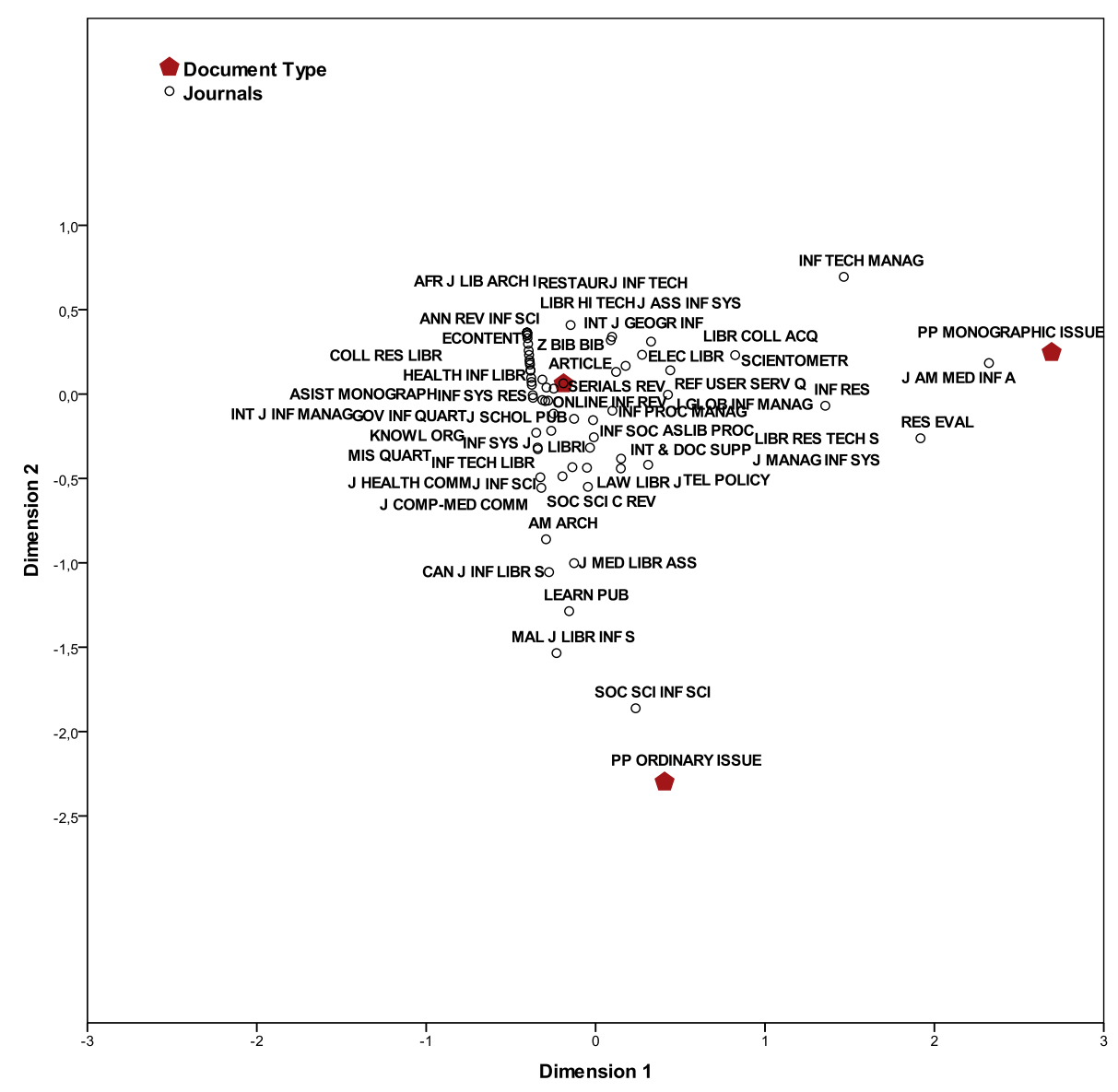

Note: the first dimension displays $88 \%$ of the inertia.

\subsection{Time span between conference date and journal publication date}

What's the time span between the holding of a conference and the publication of the related proceedings papers in a journal? It is interesting to observe that $80 \%$ of proceedings papers in LIS were published in the same year or one year after the conference (table 3). However, large differences exist between PP in ordinary and monographic issues in these regards. PP in monographic issues seem to be published faster, since $53 \%$ of them are published within the first year following the conference, while only $17 \%$ of PP in ordinary issues are published so quickly.

Table 3 and Figure 4. Distribution of PP by time span between conference and journal publication dates (WoS 1990-2008)

\begin{tabular}{|c|c|c|c|}
\hline & $\begin{array}{c}\mathrm{PP} \text { in } \\
\text { ordinary issue }\end{array}$ & $\begin{array}{c}\text { PP in } \\
\text { monographic issue }\end{array}$ & Total \\
\hline 0 year & $181 \quad(16.8 \%)$ & $1,094(52.7 \%)$ & 1,275 (40.5\%) \\
\hline 1 year & $518 \quad(48.1 \%)$ & 745 (35.9\%) & 1,263 (40.1\%) \\
\hline 2 years & $250 \quad(23.2 \%)$ & 159 (7.7\%) & 409 (13.0\%) \\
\hline 3 or more years & $128(11.9 \%)$ & $76 \quad(3.7 \%)$ & 204 (6.5\%) \\
\hline Total & $1,077(100.0 \%)$ & $2,074(100.0 \%)$ & $3,151(100.0 \%)$ \\
\hline
\end{tabular}




\subsection{Are there differences between the structure and relevance of articles and PP?}

Differences between articles and PP are explored through the study of their structure and impact by means of two different analyses. First, average values of the structural and impact indicators for articles and PP are compared through a univariate approach (comparison of means for non-parametric distributions). Secondly, a global approach is presented by means of a multivariate statistical analysis (categorical principal component analysis).

\subsubsection{Univariate analysis}

PP present a higher number of authors per document than articles, but a lower number of pages and citations per document $(p<0.001)$ (Table 4). This was observed both in the total set of documents and in the subset of journals with a higher number of PP ("core journals"). Core journals include ten journals ${ }^{3}$ with an aggregate of 8,581 papers $(20 \%$ of the total) and 2,092 proceedings papers $(66 \%$ of the total). The number of citations in the total set of journals was calculated using a variable window. Therefore, the oldest papers have more possibilities to be cited. In the core journals set, citations were calculated using a three-year citation window, and the higher number of citations received by articles as compared with PP was observed again.

Table 4. Comparative study of articles and PP: A) all journals (variable citation window); B) core journals (three-year citation window).

\begin{tabular}{|l|cc|cc|}
\hline & \multicolumn{2}{|c|}{ All journals } & \multicolumn{2}{c|}{ Core journals } \\
\cline { 2 - 5 } & Article & $\mathrm{PP}$ & $\begin{array}{c}\text { Article } \\
(\mathrm{N}=6,489)\end{array}$ & $\begin{array}{c}\mathrm{PP} \\
(\mathrm{N}=2,092)\end{array}$ \\
\hline No.Authors/doc & $1.78 \pm 1.34$ & $2.34 \pm 1.92$ & $2.24 \pm 1.76$ & $2.63 \pm 2.11$ \\
No.Centres/doc & $1.52 \pm 0.98$ & $1.64 \pm 1.18$ & $1.69 \pm 1.22$ & $1.69 \pm 1.08$ \\
No.References/doc. & $20.31 \pm 21.47$ & $20.32 \pm 22.74$ & $22.68 \pm 18.32$ & $16.60 \pm 15.90$ \\
No.Pages/doc & $11.20 \pm 8.01$ & $10.64 \pm 7.64$ & $13.14 \pm 7.57$ & $9.52 \pm 6.98$ \\
No.citations* & $4.76 \pm 16.06$ & $4.14 \pm 12.31$ & $1.74 \pm 3.76$ & $0.91 \pm 2.57$ \\
\hline
\end{tabular}

Data expressed as average +/- standard deviation.

Shaded cells show those values which are significantly higher in the article-PP comparison.

*variable citation window for all journals, three-year citation window for core journals.

However, PP form an heterogeneous set that includes PPo and PPm. PPm show a higher number of authors per document and a lower number of pages, references and citations per document than PPo $(p<0.001)$. This happens both for the total set of documents and for the set of documents published in core journals (table 5).

Table 5. Comparative study of PPo and PPm: A) all journals (variable citation window); B) core journals (three-year citation window).

\begin{tabular}{|l|cc|cc|}
\hline \multirow{2}{*}{} & \multicolumn{2}{|c|}{ All journals } & \multicolumn{2}{c|}{ Core journals } \\
\cline { 2 - 5 } & PPo & PPm & PPo & PPm \\
& $(\mathrm{N}=1,077)$ & $(\mathrm{N}=2,074)$ & $(\mathrm{N}=439)$ & $(\mathrm{N}=1,653)$ \\
\hline No.Authors/doc & $1.88 \pm 1.63$ & $2.58 \pm 2.02$ & $2.18 \pm 2.11$ & $2.75 \pm 2.09$ \\
No.Centres/doc & $1.66 \pm 1.21$ & $1.63 \pm 1.16$ & $1.86 \pm 1.37$ & $1.65 \pm 1.01$ \\
No.References/doc. & $27.47 \pm 26.43$ & $16.61 \pm 19.56$ & $25.52 \pm 22.40$ & $14.24 \pm 12.66$ \\
No.Pages/doc & $12.89 \pm 8.06$ & $9.47 \pm 7.15$ & $13.02 \pm 8.10$ & $8.59 \pm 6.33$ \\
No.citations* & $6.78 \pm 18.17$ & $2.77 \pm 7.30$ & $2.13 \pm 4.69$ & $0.62 \pm 1.57$ \\
\hline
\end{tabular}

Data expressed as average $+/$ - standard deviation.

Shaded cells show those values which are significantly higher in the PPo-PPm comparison. Statistically significant differences between PPo and PPm in all cases $(p<0.000)$, except in number of centres.

*Variable citation window for all journals, three-year citation window for core journals. 
Significant differences were found between Articles and PPm for all variables except for the number of centres both in the total journal set and in the core journals. On the contrary, articles and PPo show a more similar pattern, and even in some cases PPo show the highest values (number of references/document, in italics in table $5, p<0.01$ ). Statistically significant differences in the number of citations received by PPo and articles were not observed either in the total set of documents or in the core journal set (Figure 5).

Figure 5. Comparative study of articles, PPo and PPm by average number of centres, authors, references, pages and citations per document (limited to documents in core journals)
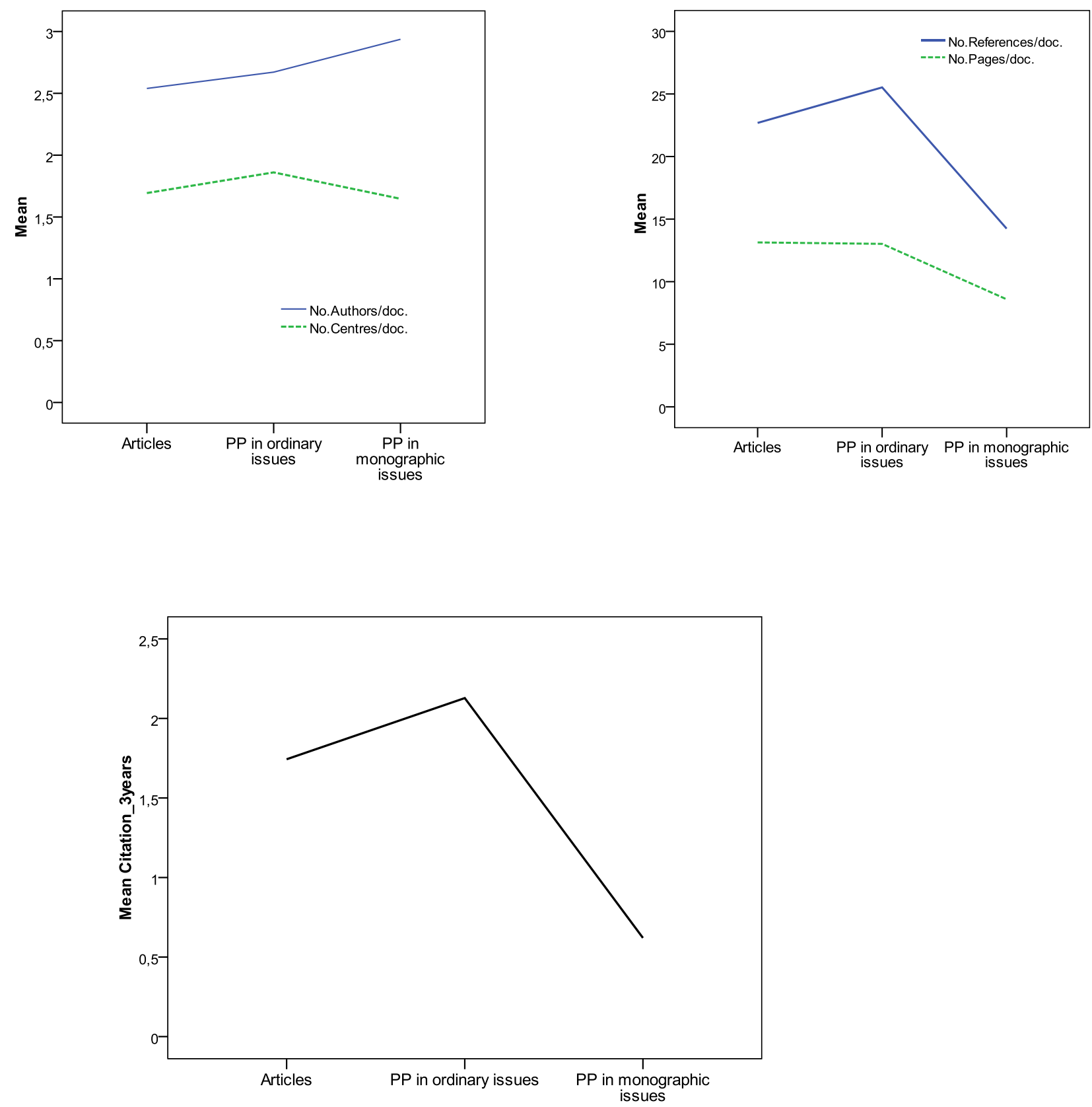
Interestingly enough, the lower impact observed for PPm published in core journals during the whole period, can be followed on a year by year basis. Figure 6 shows the citation rate of the different document types from 1990-91 to 2004-05. We can observe that articles and PP in ordinary issues obtain a very similar number of citations all over the period, while PP in monographic issues obtain substantially lower results, especially in the most recent years. The upward trend observed in the number of citations received by the three document types over the years has also been described for other scientific fields (Wallace et al., 2009) and can be partly explained by an increasing number of references per document (in our study, the average number of references per document increases from 18 in 1990-91 to 25 in 2004-05).

Figure 6. Evolution of the impact (three-year citation window) of the documents published in core journals by article and different type of PPs

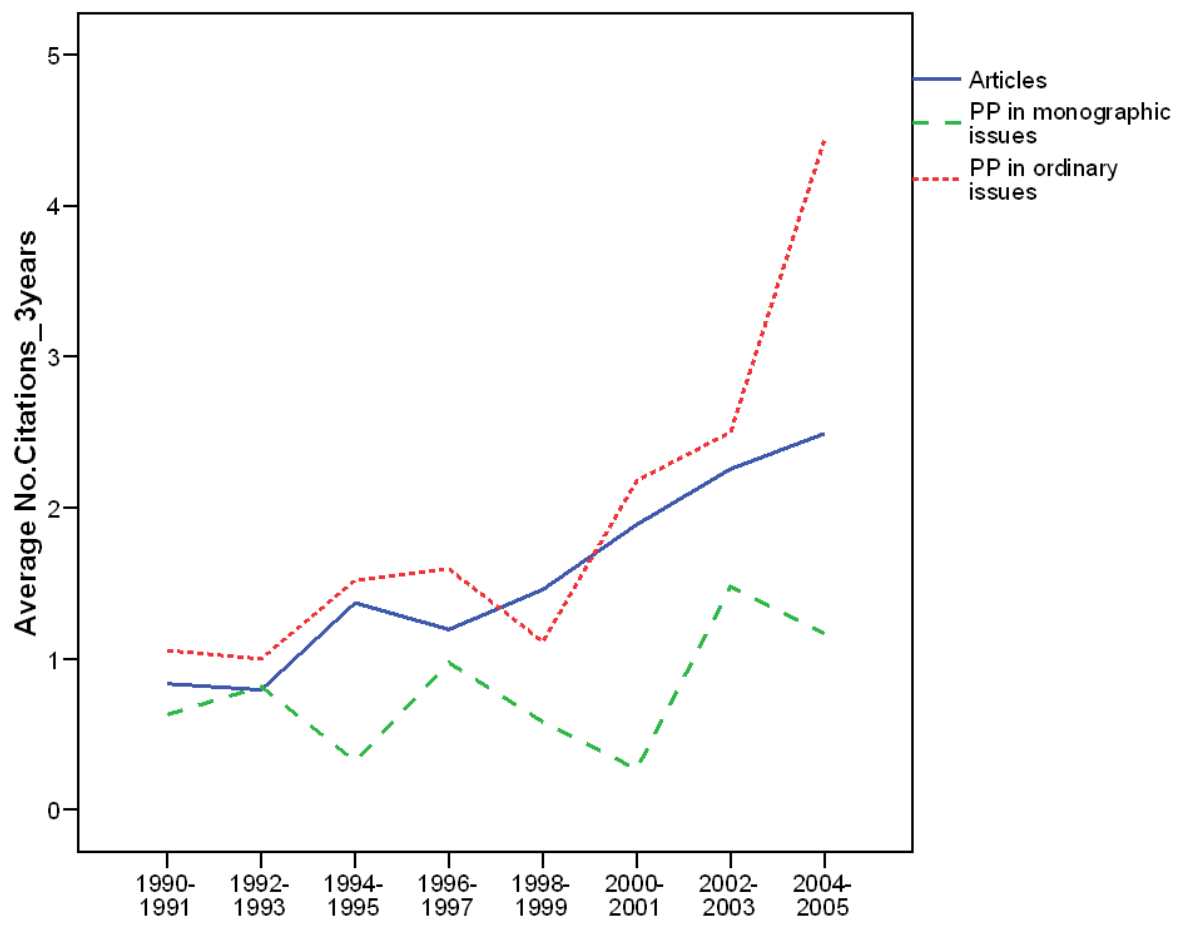

It should be noted that $15 \%$ of PPo, $11 \%$ of the articles and only $5 \%$ of PPm are highly-cited papers, defined here as those among the most-cited $10 \%\left(90^{\text {th }}\right.$ percentile). Moreover, considering the 10 most-cited papers, we can identify 8 articles and 2 PPo, while there are no PPm among these extremely cited documents.

\subsubsection{Multivariate analysis of data}

Categorical principal component analysis is a multivariate technique used in this study for the global analysis of data, since it enables us to summarise the relationship between different variables. This method can be considered as an equivalent to the standard principal component analysis to be used when some of the variables are categorical, such as document type is in the present study (three categories: articles, PPm and PPo), and was applied to the set of documents published in core journals.

As observed in figure $7 \mathrm{a}$, the system quantifies the categorical variable "document type" into a numerical one, which increases from PP in ordinary issues (-0.641), to Article $(-0.499)$ and PP in monographic issues (2.026). It is interesting to point out that PPo and articles are very close to 
each other (close values), while PPm deviate a long way from them. Therefore, the difference between PPm and the other two categories is much more important than that existing between articles and PPo.

Figure $7 \mathrm{~b}$ displays a graphic representation of the relationship between the variables. It is a twodimensional plot in which component loadings are shown as the orientation of lines along the principal axis. The first dimension separates the number of references, number of pages and number of citations by variable -which form a bundle with large positive loadings on this dimension- from the type of document. Thus, an inverse relationship between document type (on the left of the figure) and the number of references, number of pages and number of citations by variable (on the right) is observed. This means that when the document type increases (from PPo and articles -showing the lowest values- to PPm -showing the highest value-), the number of references, pages and citations tend to decrease. In other words, PPm tend to present fewer references, pages and citations. The number of authors is orthogonal (perpendicular) to the rest of the variables, so it means that it is not correlated with them. These relationships are also patent in Figure $7 \mathrm{c}$, which presents the correlation of the transformed variables: the last column shows the negative relationship between document type and the number of pages, citations and references. 
Figure 7. Categorical principal component analysis: 7a) Transformation of the variable document type. 7b) Component loadings. 7c) Correlations of the transformed variables

(a)

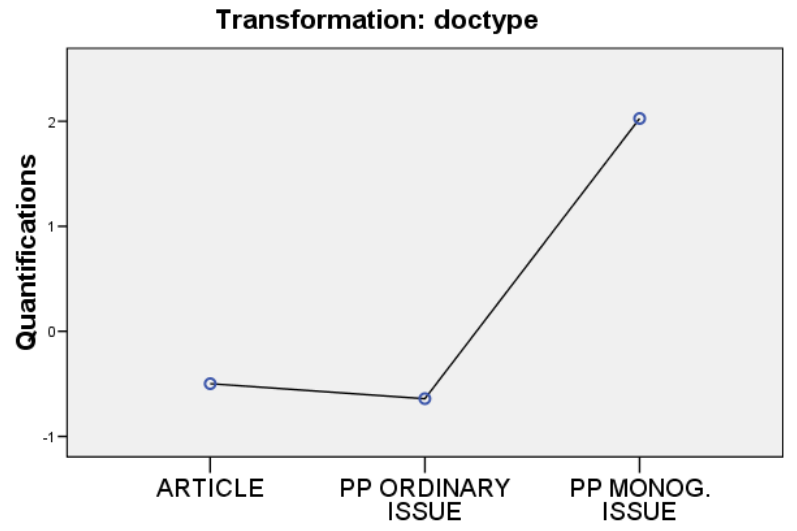

(b)

\section{Component Loadings}

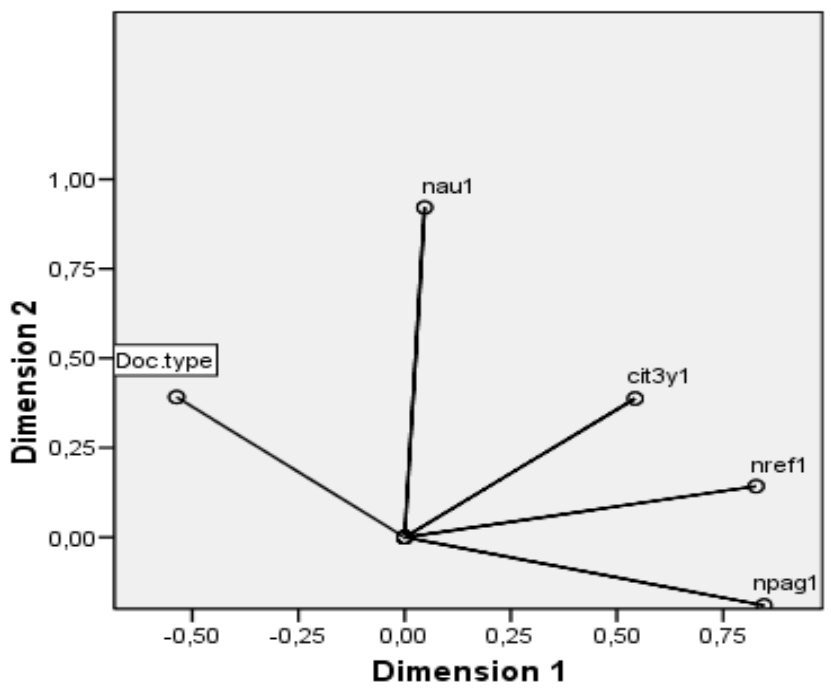

Nau1 = number of authors/document; Cit3y $1=$ number of citations/document with a three-year citation window; Nref1 $1=$ number of references/document; Npag1 $1=$ number of pages $/$ document. Cronbach's alpha $=0.86$. First dimension accounts for $62 \%$ of the variance.

(c)

\begin{tabular}{lrrrrr}
\hline & Nref1 & npag1 & \multicolumn{1}{c}{ nau1 } & \multicolumn{1}{c}{ cit3y1 } & \multicolumn{1}{c}{ doctype } \\
\hline nref1 & 1.000 & 0.608 & 0.118 & 0.211 & -0.192 \\
npag1 & & 1.000 & -0.103 & 0.195 & -0.306 \\
nau1 & & & 1.000 & 0.146 & 0.127 \\
cit3y1 & & & & 1.000 & -0.136 \\
doctype & & & & & 1.000 \\
\hline
\end{tabular}

Note: the variable number of centres was excluded because it emerged as not influential in the former analysis. 
Finally, we would like to point out that focusing on the behaviour of specific journals is beyond the scope of this paper, but differences among journals could exist and would deserve further analysis in a follow-up to this research.

\section{Discussion}

We would like to indicate some limitations of the study concerning the accuracy of some of the measures used. Firstly, proceedings papers published in ordinary journal issues can be underestimated because authors and journals do not always make explicit reference to an existing preliminary conference, as observed by Montesi and Mackenzie in their study on software engineering (2008). On the other hand, our study analyses citations received by proceedings papers in journals, but they could be underestimated if split into the original conference paper (sometimes covered by the Conference Proceedings Citation Index but not considered here) and the extended journal article. In this respect, previous studies have shown that papers in conference proceedings receive far fewer citations than journal articles, probably due to the lesser dissemination and visibility of conference proceedings (Bar-llan, 2010); consequently, we consider that our present approach can provide reliable results.

During the period under analysis, PP approximately accounted for $9 \%$ of the total number of articles in the LIS field, two-thirds of which were published in monographic issues devoted to conferences. The percentage of PP is quite irregular over the years, especially due to variations in the number of PPm. The fact is that the percentage of PP in ordinary issues remains quite stable over the years (around 3\%), while there are some publication peaks in specific years half way into our period of analysis which correspond to the publication of monographic issues (PPm). The irregularity of these data can be explained by different factors such as the periodicity of the conferences (not always held on an annual basis), changes over time in the conferences covered by the journals, changes in the journal policy regarding the type of coverage (for example, full papers or meeting abstracts; included in a supplement or in a regular journal issue) and even changes in WoS' policy regarding the coverage of proceedings papers. It should be kept in mind that only articles and proceedings papers are taken into account in this study, so conference presentations recorded by the database as meetingabstracts (only the abstract being available) are not considered here.

Our study shows that some journals, such as JAMIA or Scientometrics, tend to assemble a large share of PP. Focusing on the four PP-intensive journals (cluster 3), we have observed that they publish papers presented at different conferences strongly related with the main scope of the journal. That is the case of JAMIA, official journal of the American Medical Informatics Association, which covers -among others- papers presented at the Annual Symposium on American-Medical-Informatics-Association in specific years; Information Research, which covers proceedings papers of the Information Seeking in context Conference, or Research Evaluation, which covers papers presented at the Conference on Science and Technology Indicators. Publication journals of PP are usually "core" titles in the conference subject, and their journal editors -usually renowned scientists in the field- can also be conference participants with varying degrees of involvement (active presenter, member of scientific committee, etc.).

Among the advantages of the publication of special monographic journal issues devoted to a conference it is worth mentioning that it contributes to disseminate the main research developments in the field as well as to increase the visibility of the conference. Accordingly, this practice can be advantageous for the conference, but also for the discipline and for the authors themselves. Concerning the discipline, the monographic issue provides an interesting overview of cutting-edge research in the field and it displays the state-of-the-art in the speciality. As for the authors, they can get their papers published faster than following the standard publication process in journals (Montesi and Mackenzie, 2008). In spite of these positive effects, the publication of a special journal issue devoted to a conference implies a great deal of work for both conference organisers and journal editors, a significant part of which is the review process of papers required to guarantee the quality of the final product. 


\subsection{Do articles and PP differ in their structure and impact?}

In principle, the quality of proceedings papers is guaranteed by the fact that they have passed through two different refereeing processes: first at the conference and later at the journal. However, our results show that there are significant differences between articles and PP in relation to the structure and impact of their research: PP display a higher number of authors per document, maybe related to the need of scientists to be authors or co-authors to attend the conference, while articles show a higher number of pages and receive a higher number of citations, which point to a more complete and influential research (Question 1).

However, differences between PPo and PPm are observed in this study (Question 2). PP in ordinary issues are quite similar to standard articles in the structure and impact of the research, while PP in monographic issues are less comprehensive (fewer pages and references) and tend to receive fewer citations. Can this lower impact be associated to a less rigorous evaluation process? The faster publication of PPm as compared to PPo might support this hypothesis (Question 3), especially because $50 \%$ of the PPm were published during the same year in which the conference was held. This rate of publication is faster than both that observed for PPo in our study (17\% in the conference year) and that described in the literature for articles derived from presentations at different ISSI Conferences (less than 30\% of the papers were published in the conference year) (Aleixandre et al., 2009). In the latter study we cannot discriminate between PPo and PPm, but it is interesting to underline that most papers were published the following year after the conference.

An outstanding result is the fact that PP in ordinary issues tend to surpass standard articles in some features such as number of references, which suggest a more complete revision and discussion of related literature. Moreover, although the differences between standard articles and PPo in the average number of citations are not statistically significant, it is noticeable that the presence of PPo among highly-cited documents is higher than expected (15\% of the PPo vs. $11 \%$ of the articles and $5 \%$ of the PPm are highly-cited papers). The fact that PPo take longer to be published than PPm can be a crucial factor, since authors may have longer time to revise the paper, which can be improved with colleague's suggestions during the conference and might result in a more solid final article.

\subsection{Implications of the research}

Some implications can be drawn from our research with interest for different sectors of the scientific community, including bibliometricians, research evaluators and scientists themselves.

Firstly, we conclude that differences in the relevance of documents based only in their WoS document type (article or PP) should not be inferred. PP are an heterogeneous set which comprises PPm, on average less comprehensive and influential than articles; but also PPo, which tend to be more complete papers -according to the number of references- and receive as many citations as articles. This finding is interesting for scientists, who have to choose what document types to read from an increasing number of papers; but also for bibliometricians, who select the most valuable document types for their studies; or for research evaluators, who may assess or even weigh differently the papers of scientists by document type.

On the other hand, the function and value of post-conference monographic issues is called into question here. In principle, the main function of monographic issues devoted to a conference is contributing to disseminate current research and provide a global view of the state-of-the-art in the speciality. Compared with Conference Proceedings Books, the publication of extended articles can provide some added value: (a) making full papers available (sometimes only abstracts are included in conference proceedings); (b) improving the visibility of research (in general, journals are more visible than proceedings books); (c) selection of the "best" papers which are able to pass a journal review process. In our study, objective $b$ is accomplished in all cases, but we do not know to what extent PP correspond to papers only available as conference abstracts before publication (objective a) or if PP have passed through a review process to decide on publication (objective $\mathrm{c}$ ). The latter seems to be essential to guarantee the highest interest and relevance of the final journal issue. 
The under-average impact of the final PPm and the short time span between the holding of the conference and the PPm publication suggest that they have passed, on average, a very slight review process. To what extent are authors doing good use of one of the conference functions which is taking advantage of participants' feedback on their presentations to improve final papers? To what extent are proceedings papers being thoroughly reviewed? We do not know the answers to these questions, but we consider that a good review of proceedings papers is needed to maximise the added value of PPm as compared with Proceedings Books.

All in all, it is clear that the editorial procedures followed in the publication of proceedings papers (and the rigour of the paper revision, if any) may vary from one journal to another, so it would be interesting to conduct a comparative study of PP and articles at journal level, as well as to analyse how it evolves over time. Journal editors may be especially interested in this type of study, since the publication of low-quality PPm could reduce the prestige of the publication journal and even have an adverse effect on its impact factor ${ }^{4}$.

Finally, this study offers a global overview of the LIS field, but due to inter-field differences in the scholarly communication process, the results cannot be generalised to other disciplines. Further analyses dealing with other scientific fields would be needed.

\section{References}

Aleixandre-Benavent, R.; Gonzalez-Alcaide, G.; Miguel-Dasit, A.; Navarro-Molina, C.; Valderrama-Zurián, J.C. (2009) Full-text publications in peer-reviewed journals derived from presentations at three ISSI Conferences. Scientometrics, 80(2), 407-418.

Bar-Ilan, J. (2010) Web of Science with the Conference Proceedings Citation Indexes: the case of computer science. Scientometrics, 83(3), 809-824.

Bordons, M.; Gómez, I. (2000) Collaboration networks in Science. In: Cronin, B. \& Atkins, H.B. (Eds.). The web of knowledge: A Festschrift in honor of Eugene Garfield (pp. 197-213) Medford, N.J.: Information Today, Inc. \& American Society for Information Science.

Miguel-Dasit, A.; Marti-Bonmati, L.; Sanfeliu, P.; Aleixandre, R. (2006) Scientific papers presented at the European Congress of Radiology 2000: publication rates and characteristics during the period 2000-2004. European Radiology, 16(2), 445-450.

Drott, M.C. (1995) Reexamining the role of conference papers in scholarly communication. Journal of the American Society for Information Science, 46(4), 299-305.

Glanzel, W.; Schlemmer, B.; Schubert, A.; Thijs, B. (2006) Proceedings literature as additional data source for bibliometric analysis. Scientometrics, 68(3), 457-473.

Goodrum, AA.; MacCain, K.W.; Lawrence, S.; Giles, C.L. (2001) Scholarly publishing in the Internet age: a citation analysis of computer science literature. Information Processing and Management, 37(5), 661-675.

Haslam, N.; Ban, L.; Kaufmann, L.; Loughnan, S.; Peters, K.; Whelan, J. ; Wilson, S. (2008) What makes an article influential? Predicting impact in social and personality psychology. Scientometrics, 76(1), 169-185.

Kho, M.E.; Brouwers, M.C. (2009) Conference abstracts of a new oncology drug do not always lead to full publication: proceed with caution. Journal of Clinical Epidemiology, 62, 752-758.

Lisée, C.; Lariviére, V.; Archambault, E. (2008) Conference proceedings as a source of scientific information: a bibliometric analysis. Journal of the American Society for Information Science and Technology, 59(11), 1776-1784.

Meulman, J.J., Heiser, W.J., \& SPSS Inc. (2008). SPSS Categories 18, Chapter 3. Chicago, IL:SPSS 
http://support.spss.com/productsext/statistics/documentation/18/client/User\%20Manuals/English IPASW\%20Categories\%2018.pdf

Why the number of articles in Web of Science has gone down and the number of proceedings papers has gone up. http://isiwebofknowledge.com/products tools/multidisciplinary/webofscience/cpci/

McVeigh, M.E.; Mann, S.J. (2009) The journal impact factor denominator. Defining citable (counted) items. JAMA, 302(10), 1107-1109.

Moed, H.F. Citation analysis in research evaluation. Dordrecht, The Netherlands: Springer, 2005.

Montesi, M.; Mackenzie Owen, J. (2008) From Conference to Journal publication: how conference papers in software engineering are extended for publication in journals. Journal of the American Society for Information Science and Technology, 59(5), 816-829.

Wallace, M.L.; Larivière, V.; Gingras, Y. (2009) Modeling a century of citation distributions. Journal of Informetrics, 3(4), 296-303.

Web of Science (2009). Using the proceedings paper document type. Retrieve may 8, 2010. In: http://isiwebofknowledge.com/products tools/multidisciplinary/webofscience/cpci/usingproceedi ngs/

Acknowledgments. We are grateful to our colleagues from the ACUTE team and to Laura Barrios and J. Manuel Rojo for their valuable help in the statistical analysis of data. This research was developed in the framework of CSIC's research project 200410E605.

Annex 1. Articles and proceedings papers in monographic and ordinary issues (PPm and PPo) by journals (WoS, 1990-2008) (in descending order of number of total PP)

\begin{tabular}{|c|c|c|c|c|c|c|c|c|c|}
\hline \multirow{2}{*}{ Journal } & \multicolumn{4}{|c|}{ Number } & \multicolumn{3}{|c|}{$\%$} & \multicolumn{2}{|c|}{ Total PP } \\
\hline & Articles & PPm & Ppo & Total & Articles & PPm & Ppo & No. & $\%$ \\
\hline J AMER MED INFORM ASSOC & 1045 & 867 & 94 & 2006 & 52.09 & 43.22 & 4.69 & 961 & 47.91 \\
\hline SCIENTOMETRICS & 1325 & 329 & 42 & 1696 & 78.13 & 19.40 & 2.48 & 371 & 21.88 \\
\hline TELECOMMUN POLICY & 739 & 91 & 57 & 887 & 83.31 & 10.26 & 6.43 & 148 & 16.69 \\
\hline SOC SCI INFORM & 384 & 35 & 83 & 502 & 76.49 & 6.97 & 16.53 & 118 & 23.51 \\
\hline ELECTRON LIBR & 676 & 90 & 10 & 776 & 87.11 & 11.60 & 1.29 & 100 & 12.89 \\
\hline INF RES & 179 & 73 & 14 & 266 & 67.29 & 27.44 & 5.26 & 87 & 32.71 \\
\hline INFORM PROCESS MANAGE & 1028 & 41 & 44 & 1113 & 92.36 & 3.68 & 3.95 & 85 & 7.64 \\
\hline RES EVALUAT & 108 & 69 & 14 & 191 & 56.54 & 36.13 & 7.33 & 83 & 43.46 \\
\hline ASLIB PROC & 615 & 51 & 27 & 693 & 88.74 & 7.36 & 3.90 & 78 & 11.26 \\
\hline LEARN PUBL & 390 & 7 & 54 & 451 & 86.47 & 1.55 & 11.97 & 61 & 13.53 \\
\hline INTERLEND DOC SUPPLY & 337 & 30 & 25 & 392 & 85.97 & 7.65 & 6.38 & 55 & 14.03 \\
\hline SOC SCI COMPUT REV & 424 & 21 & 33 & 478 & 88.70 & 4.39 & 6.90 & 54 & 11.30 \\
\hline LAW LIBR J & 440 & 22 & 30 & 492 & 89.43 & 4.47 & 6.10 & 52 & 10.57 \\
\hline J INFORM SCI & 732 & 0 & 48 & 780 & 93.85 & 0.00 & 6.15 & 48 & 6.15 \\
\hline INT J GEOGR INF SCI & 490 & 42 & 5 & 537 & 91.25 & 7.82 & 0.93 & 47 & 8.75 \\
\hline J MED LIBR ASSOC & 324 & 9 & 37 & 370 & 87.57 & 2.43 & 10.00 & 46 & 12.43 \\
\hline J MANAGE INFORM SYST & 289 & 26 & 20 & 335 & 86.27 & 7.76 & 5.97 & 46 & 13.73 \\
\hline LIBRI & 414 & 25 & 19 & 458 & 90.39 & 5.46 & 4.15 & 44 & 9.61 \\
\hline J INFORM TECHNOL & 336 & 34 & 8 & 378 & 88.89 & 8.99 & 2.12 & 42 & 11.11 \\
\hline INFORM TECHNOL LIBR & 438 & 10 & 30 & 478 & 91.63 & 2.09 & 6.28 & 40 & 8.37 \\
\hline LIBR RESOUR TECH SERV & 350 & 21 & 19 & 390 & 89.74 & 5.38 & 4.87 & 40 & 10.26 \\
\hline J HEALTH COMMUN & 381 & 13 & 25 & 419 & 90.93 & 3.10 & 5.97 & 38 & 9.07 \\
\hline LIBR COLLECT ACQUIS & 247 & 30 & 5 & 282 & 87.59 & 10.64 & 1.77 & 35 & 12.41 \\
\hline J AM SOC INF SCI TECHNOL & 1018 & 0 & 29 & 1047 & 97.23 & 0.00 & 2.77 & 29 & 2.77 \\
\hline INFORM SOC & 255 & 14 & 15 & 284 & 89.79 & 4.93 & 5.28 & 29 & 10.21 \\
\hline J AMER SOC INFORM SCI & 840 & 9 & 18 & 867 & 96.89 & 1.04 & 2.08 & 27 & 3.11 \\
\hline INT J INFORM MANAGE & 558 & 5 & 17 & 580 & 96.21 & 0.86 & 2.93 & 22 & 3.79 \\
\hline
\end{tabular}




\begin{tabular}{|c|c|c|c|c|c|c|c|c|c|}
\hline \multirow{2}{*}{ Journal } & \multicolumn{4}{|c|}{ Number } & \multicolumn{3}{|c|}{$\%$} & \multicolumn{2}{|c|}{ Total PP } \\
\hline & Articles & PPm & Ppo & Total & Articles & PPm & Ppo & No. & $\%$ \\
\hline GOVT INFORM QUART & 478 & 7 & 15 & 500 & 95.60 & 1.40 & 3.00 & 22 & 4.40 \\
\hline RESTAURATOR & 229 & 20 & 2 & 251 & 91.24 & 7.97 & 0.80 & 22 & 8.76 \\
\hline J ACAD LIBR & 935 & 0 & 20 & 955 & 97.91 & 0.00 & 2.09 & 20 & 2.09 \\
\hline MIS QUART & 405 & 0 & 18 & 423 & 95.74 & 0.00 & 4.26 & 18 & 4.26 \\
\hline Z BIBL BIBLIOGR & 376 & 8 & 10 & 394 & 95.43 & 2.03 & 2.54 & 18 & 4.57 \\
\hline REF USER SERV Q & 317 & 10 & 8 & 335 & 94.63 & 2.99 & 2.39 & 18 & 5.37 \\
\hline J SCHOLARLY PUBL & 264 & 4 & 12 & 280 & 94.29 & 1.43 & 4.29 & 16 & 5.71 \\
\hline AM ARCHIVIST & 166 & 0 & 16 & 182 & 91.21 & 0.00 & 8.79 & 16 & 8.79 \\
\hline CAN J INFORM LIB SCI & 141 & 0 & 16 & 157 & 89.81 & 0.00 & 10.19 & 16 & 10.19 \\
\hline PROGRAM-ELECTRON LIB & 264 & 5 & 10 & 279 & 94.62 & 1.79 & 3.58 & 15 & 5.38 \\
\hline ONLINE INF REV & 355 & 5 & 9 & 369 & 96.21 & 1.36 & 2.44 & 14 & 3.79 \\
\hline LIBR HI TECH & 322 & 14 & 0 & 336 & 95.83 & 4.17 & 0.00 & 14 & 4.17 \\
\hline J COMPUT-MEDIAT COMMUN & 198 & 0 & 14 & 212 & 93.40 & 0.00 & 6.60 & 14 & 6.60 \\
\hline KNOWL ORGAN & 230 & 0 & 12 & 242 & 95.04 & 0.00 & 4.96 & 12 & 4.96 \\
\hline J ASSOC INF SYST & 64 & 10 & 2 & 76 & 84.21 & 13.16 & 2.63 & 12 & 15.79 \\
\hline LIBR QUART & 256 & 3 & 8 & 267 & 95.88 & 1.12 & 3.00 & 11 & 4.12 \\
\hline INFORM SYST J & 214 & 0 & 11 & 225 & 95.11 & 0.00 & 4.89 & 11 & 4.89 \\
\hline INFORM TECHNOL MANAG & 23 & 10 & 0 & 33 & 69.70 & 30.30 & 0.00 & 10 & 30.30 \\
\hline LIBR TRENDS & 763 & 0 & 9 & 772 & 98.83 & 0.00 & 1.17 & 9 & 1.17 \\
\hline SERIALS REV & 78 & 7 & 2 & 87 & 89.66 & 8.05 & 2.30 & 9 & 10.34 \\
\hline J GLOB INF MANAG & 46 & 7 & 2 & 55 & 83.64 & 12.73 & 3.64 & 9 & 16.36 \\
\hline INFORM MANAGEMENT & 1043 & 0 & 8 & 1051 & 99.24 & 0.00 & 0.76 & 8 & 0.76 \\
\hline LIBR INFORM SCI RES & 353 & 0 & 8 & 361 & 97.78 & 0.00 & 2.22 & 8 & 2.22 \\
\hline INFORM SYST RES & 293 & 0 & 8 & 301 & 97.34 & 0.00 & 2.66 & 8 & 2.66 \\
\hline J DOC & 426 & 0 & 7 & 433 & 98.38 & 0.00 & 1.62 & 7 & 1.62 \\
\hline COLL RES LIBR & 629 & 0 & 6 & 635 & 99.06 & 0.00 & 0.94 & 6 & 0.94 \\
\hline LIBR J & 2144 & 0 & 5 & 2149 & 99.77 & 0.00 & 0.23 & 5 & 0.23 \\
\hline SCIENTIST & 3676 & 0 & 4 & 3680 & 99.89 & 0.00 & 0.11 & 4 & 0.11 \\
\hline J LIBR INF SCI & 291 & 0 & 4 & 295 & 98.64 & 0.00 & 1.36 & 4 & 1.36 \\
\hline MALAYS J LIBR INF SC & 19 & 0 & 3 & 22 & 86.36 & 0.00 & 13.64 & 3 & 13.64 \\
\hline HEALTH INFO LIBR J & 156 & 0 & 2 & 158 & 98.73 & 0.00 & 1.27 & 2 & 1.27 \\
\hline ONLINE & 1070 & 0 & 1 & 1071 & 99.91 & 0.00 & 0.09 & 1 & 0.09 \\
\hline PORTAL-LIBR ACAD & 210 & 0 & 1 & 211 & 99.53 & 0.00 & 0.47 & 1 & 0.47 \\
\hline J INFORMETR & 62 & 0 & 1 & 63 & 98.41 & 0.00 & 1.59 & 1 & 1.59 \\
\hline ASIST MON SER & 51 & 0 & 1 & 52 & 98.08 & 0.00 & 1.92 & 1 & 1.92 \\
\hline ECONTENT & 533 & 0 & 0 & 533 & 100.00 & 0.00 & 0.00 & 0 & 0.00 \\
\hline PROF INF & 185 & 0 & 0 & 185 & 100.00 & 0.00 & 0.00 & 0 & 0.00 \\
\hline LIBR INFORM SC & 110 & 0 & 0 & 110 & 100.00 & 0.00 & 0.00 & 0 & 0.00 \\
\hline AFR J LIBR ARCH & 31 & 0 & 0 & 31 & 100.00 & 0.00 & 0.00 & 0 & 0.00 \\
\hline ANNU REV INFORM SCI TECH & 29 & 0 & 0 & 29 & 100.00 & 0.00 & 0.00 & 0 & 0.00 \\
\hline INVESTIG BIBLIOTECOL & 25 & 0 & 0 & 25 & 100.00 & 0.00 & 0.00 & 0 & 0.00 \\
\hline REV ESP DOC CIENT & 24 & 0 & 0 & 24 & 100.00 & 0.00 & 0.00 & 0 & 0.00 \\
\hline INF TARSAD & 19 & 0 & 0 & 19 & 100.00 & 0.00 & 0.00 & 0 & 0.00 \\
\hline Total & 31865 & 2074 & 1077 & 35016 & & 5.92 & 3.08 & 3151 & 9.00 \\
\hline
\end{tabular}

Note: percentages are calculated in rows.

\section{Footnotes}

1. Preliminary results of this research were presented at STI Conference 2010 held in Leiden.

2. Including Science Citation Index Expanded (SCIE), Social Sciences Citation Index (SSCI) and Arts and Humanities Citation index (AHCl).

3. Journal of the American Medicine Informatics Association, Scientometrics, Telecommunications Policy, Social Science Information sur les Sciences Sociales, Electronic Library, Information Research, Information Processing \& Management, Research Evaluation, Aslib Proceedings and Learned Publishing.

4. As an example we can mention the case of JAMIA, which moved in the journal ranking in descending order of impact factor from the $24^{\text {th }}$ position in 2002 (IF=0.541) to the third position in $2003(\mathrm{IF}=2.510)$, after removing articles in special issues from the calculation of the impact factor. 